\title{
MAG-DHA, precursor of D-series resolvins, induces powerful resolution of various components of pulmonary hypertension induced by monocrotaline in rats
}

\author{
Roddy Hiram ${ }^{1}$, Caroline Morin ${ }^{1,2}$, Samuel Fortin ${ }^{2}$ and Eric Rousseau ${ }^{*}$ \\ ${ }^{1}$ Department of obstetrics-gynecology, Faculty of Medicine and Health Sciences, Université de Sherbrooke, Sherbrooke, QC, Canada \\ ${ }^{2} \mathrm{SCF}$ Pharma, Ste-Luce, QC, Canada
}

\begin{abstract}
Pulmonary hypertension $(\mathrm{PH})$ is a disease of the lung vessels involving abnormal vasoconstriction which limits lung blood circulation and oxygenation. Despite current pharmacological treatments, $\mathrm{PH}$ still remains associated with high morbidity. Our hypothesis is that systemic lung inflammation may be resolved via relevant treatment in a rat model of Monocrotaline (MCT)-induced PH. The goal of the study was to assess the resolving effects of monoacylglyceride docosahexaenoic acid (MAG-DHA) on MCT-induced PH by measuring inflammation biomarkers and contractility levels in pulmonary arteries. Experimental rats were administered $60 \mathrm{mg} / \mathrm{kg}$ of MCT on day 1 and subsequently treated from day 14 onward with $231 \mathrm{mg} / \mathrm{kg}$ MAG-DHA daily for 7 days and compared with non-treated MCT animals and untreated controls. Results show that MAG-DHA normalized right ventricle (RV) weight which was significantly increased in the MCT-treated group compared to controls. Mean artery wall thickness increased from $12 \mu \mathrm{m}$ in control rats to $48 \mu \mathrm{m}$ in MCT rats while MAG-DHA treatment significantly curbed this increase with a resulting wall thickness of $20 \mu \mathrm{m}$. MCT rats also displayed increased arterial reactivity in response to $30 \mathrm{nM} \mathrm{U}-46619$ (thromboxane A2 analog) and $1 \mu \mathrm{M}$ PDBu (a potent PKC activator), whereas MAG-DHA treatment significantly decreased this pharmacological hyper-responsiveness. Western blot analysis confirmed that MCT treatment increased the phosphorylation levels of P-CPI-17 and P-MYPT-1, which were largely reversed after MAG-DHA treatment. Lastly, under MCT condition, a large array of inflammatory biomarkers were enhanced, including TNF- $\alpha$, COX-2, STAT-3 as well as the phosphorylated nuclear factors $\mathrm{P}-\mathrm{C}-\mathrm{Fos}, \mathrm{P}-\mathrm{C}-\mathrm{Jun}$ and $\mathrm{P}-\mathrm{NF}-\kappa \mathrm{B}$, with MAG-DHA treatment strongly reversing this inflammatory profile. In conclusion, a 7-day treatment with MAG-DHA is able to resolve the inflammatory status in a rat $\mathrm{PH}$ model.
\end{abstract}

\section{Introduction}

Pulmonary hypertension $(\mathrm{PH})$ is a rare and progressive disease that affects numerous individuals without distinction of age, gender or socio-economic status [1]. In most instances, patients are unaware that they are sick since the early stages of this disease are characterized by symptoms that can be confused with benign diseases such as influenza or light fever [2]. When the diagnosis of $\mathrm{PH}$ is ultimately confirmed, the disease is generally at its most severe stage and described as irreversible [3].

In order to better understand the disease and perform appropriated therapeutic approach, a classification of $\mathrm{PH}$ has been proposed from the $4^{\text {th }}$ PH World Symposium of Dana Point in California 2008 [4]. 5 groups of $\mathrm{PH}$ are identified. Group 1 is pulmonary hypertension with idiopathic cause, heritable, induced by toxics and drugs, associated with infection such as HIV, persistent $\mathrm{PH}$ of the newborn. Group 2 is $\mathrm{PH}$ due to left heart diseases. Group 3 is $\mathrm{PH}$ due to lung diseases and hypoxemia. Group 4 is chronic thromboembolic pulmonary hypertension. Group 5 is $\mathrm{PH}$ with multifactorial origins.

From a pathophysiological standpoint, $\mathrm{PH}$ is characterized by a substantial dysfunction of the endothelium which is severely disorganized and hypertrophied. Abnormal expression and secretion of ET-1 by the endothelium leads to over-proliferation and reactivity of pulmonary artery smooth muscle (PASM) cells [5]. The adventitia of the artery wall is the site of significant inflammation, due to the secretion of cytokines and chemokines such as IL-1 $\beta$, IL-6, IL-13, TNF- $\alpha$, CCL5/
RANTES, CXCL10 and accumulation of pro-inflammatory cells such as dendritic cells, $\mathrm{T}$ and $\mathrm{B}$ lymphocytes, mast cells and macrophages [6]. This phenomenon contributes to an increase in paracrine signaling pathways and leads to vasoconstriction of the pulmonary arteries.

Moreover, in the media of the pulmonary artery wall, PASM cells have been described to express specific biomarkers of $\mathrm{PH}$ such as TMEM16A (Transmembrane member $16 \mathrm{~A}$ ), a voltage sensitive calcium-activated chloride channel [7]. PASM cells also present increased $\mathrm{Ca}^{2+}$ sensitivity and hyperreactivity in response to pharmacological agents. Among these, endothelin-1, U-46619 (an agonist of TP receptors) and $\mathrm{PDBu}$, a PC activator, are involved in the phosphorylation of CPI-17 (C-kinase-activated protein phosphatase-1 inhibitor of $17 \mathrm{kDa}$ ), which in turn contributes in maintaining the myosin light chain phosphatase in its phosphorylated (inactivated) form consistent with the phosphorylation of myosin phosphatase target subunit 1 -P-MYPT-1- [8]. This impedes the dephosphorylation

Correspondence to: Eric Rousseau, $\mathrm{PhD}$, Department of obstetrics-gynecology, Faculty of Medicine and Health Sciences, Université de Sherbrooke, 3001, $12^{\text {th }}$ avenue north, J1H 5N4, Sherbrooke, QC, Canada, Tel: 819-346 1110; 819-347 5306 ; Fax: 819-564-5399; E-mail: Eric.Rousseau@USherbrooke.ca

Key words: D-series resolvins, docosahexaenoic acid monoacylglyceride, inflammation, pulmonary hypertension

Received: April 12, 2016; Accepted: May 06, 2016; Published: May 11, 2016 
Hiram R (2016) MAG-DHA, precursor of D-series resolvins, induces powerful resolution of various components of pulmonary hypertension induced by monocrotaline in rats

of myosin light chain and the maintenance of tone in PASM cells and media layer [9].

Instead of the typical $10 \mathrm{mmHg}$ at rest in normal subjects, patients with $\mathrm{PH}$ display mean pulmonary arterial blood pressure reaching 25 $\mathrm{mmHg}[10]$. However the role of inflammation within the pulmonary artery wall has neither been comprehensively addressed nor adequately treated. Hence the resolution of these inflammatory processes may be of prime clinical interest.

Over the last decade, various treatments have been proposed to improve patients' lives. Most target endothelial cell pathways that potentially lead to vasodilatation, such as the use of prostacyclin analogs therapy [11], NO production [2], PDE-5 inhibition [3] or activation of atrial natriuretic peptide pathways, in order to tackle the negative effects of ET-1 [11]. Regrettably, none of these treatments are able to cure patients with $\mathrm{PH}$ and the disease ultimately continues its evolution into right ventricular hypertrophy, heart failure and death [12].

The goal of the present study was to assess the functional consequences of pulmonary artery wall inflammation in-vivo as well as the putative effects of pro-resolving agents on various inflammatory processes.

Accordingly, several omega-3 derivatives have been identified in various physiological systems as pro-resolving compounds $[8,13,14]$, able to speed up resolution of inflammation as attested by several reports $[15,16]$. Our group has already investigated the impact of different monoacylglyceride compounds and shown that monoacylglyceride docosahexaenoic acid (MAG-DHA) [15], monoacylglyceride docosapentaenoic acid (MAG-DPA) [16], and D-series resolvins such as Resolvin D1 were able to abolish the pro-inflammatory effects of TNF- $\alpha$ and IL- 6 as well as the induced hyperreactivity and calcium sensitivity in human pulmonary arteries [17].

Our current hypothesis is that per os administrations of MAG-DHA can potentially normalize the pro-inflammatory responses and thus restore pulmonary artery reactivity due to a decrease in inflammation as revealed by changes in specific biomarkers. The present study shows that a 7-day treatment of daily per os administration of $231 \mathrm{mg} / \mathrm{kg}$ of MAG-DHA fourteen days after a single intraperitoneal injection of 60 $\mathrm{mg} / \mathrm{kg}$ Monocrotaline (MCT) was able to reverse and normalize key histological, cellular, molecular and inflammatory markers of MCTinduced pulmonary hypertension disease in rodents.

\section{Material and methods}

\section{Synthesis of n-3 PUFA monoacylglycerides}

MAG-DHA was synthesized using ethyl ester as starting material, as previously described [18]. The resulting molecule was comprised of DHA attached in the sn-1 position of glycerol. MAG-DHA was synthesized and purified by SCF-Pharma (Rimouski, Quebec, Canada) and was kindly provided by Dr Samuel Fortin.

\section{Drugs and chemical reagents}

U-46619 was obtained from Cayman Chemical (Ann Arbor, Michigan). PDBu, Anti-CPI-17, anti-phospho-CPI-17, anti-phosphoc-Jun (Ser63), anti-phospho-c-Fos (Ser32), anti-Cox-2, anti-NF- $\mathrm{B}$ and anti-phospho-NF- $\mathrm{kB}$ were purchased from Cedarlane (Burlington, Ontario, Canada). Anti-TNF- $\alpha$ and anti-TMEM16A were purchased from Abcam (Toronto, Ontario, Canada). Antibodies against MYPT-1 and P-MYPT-1 (phosphorylated form of MYPT-1 on Thr 696) were obtained from Santa Cruz Biotechnology (California, United States). Crotaline (Monocrotaline), Type IV Collagenase and Type IV Elastase were purchased from Sigma-Aldrich (St. Louis, MO, United States). DMEM/F-12, penicillin-streptomycin and FBS were obtained from Gibco Invitrogen (Burlington, Ontario, Canada).

\section{Ethics Committee Approval}

The current study involving rats was approved by our institution's Ethics Committee under protocol No. 018-12, which respects the high standards for animal ethics and care in science of the Canadian Council on Animal Care (CCAC). According to the Ethics Committee guidelines, animals were respectfully and painlessly used, only to contribute to the development of scientific knowledge for the benefit of human and animal health.

\section{Animal model of pulmonary hypertension (PH)}

Male Wistar rats weighting 250-275 g were acclimated for 7 days prior to the start of the experiments. Rats were randomly assigned into three groups: control (untreated) group, monocrotaline (MCT)treated group which received one $60 \mathrm{mg} / \mathrm{kg}$ intraperitoneal injection of monocrotaline, and the MCT + MAG-DHA group in which MCTinjected rats were treated for 7 days (from D14 to D21) with a daily per os administration of $231 \mathrm{mg} / \mathrm{kg}$ of MAG-DHA) (Figure 1A). The control groups received equivalent doses of vehicle. The oral dose of $231 \mathrm{mg} / \mathrm{kg}$ was chosen in order to obtain the maximum human equivalent allowed by Health Canada which is $3.0 \mathrm{~g} /$ day according to Health Canada Draft Guidelines [19]. The established MCT-induced $\mathrm{PH}$ model follows the protocol previously described by several groups $[15,20,21]$.

At day 22, all rats were euthanized with pentobarbital sodium. Blood was collected by cardiac puncture and hearts and lungs were excised. Right ventricle (RV) weight was measured and compared to the weight of the left ventricle (LV) plus ventricular septum (S). Fulton's index was determined as the weight ratio of RV/ LV $+\mathrm{S}$. This index allows the assessment of the induced RV hypertrophy as a result of MCT injection and pulmonary artery wall hypertrophy. Lung arteries were dissected and isometric tension measurements were performed as described below. Pulmonary arteries were also used to isolate smooth muscle cells. Histological analysis of pulmonary artery wall thickness has been performed to determine an average size. Then, media thickness from MCT and MCT + MAG-DHA treated-animals were compared to the media thickness of the control group to assess putative increase. Furthermore, homogenate preparations were performed on lung tissues exactly as previously described [16].

\section{Isolation of pulmonary arterial smooth muscle (PASM) cells}

Second order pulmonary artery (PA) rings were carefully dissected from rat lungs and incubated for $1 \mathrm{~h}$ at $37^{\circ} \mathrm{C}, 5 \% \mathrm{CO}_{2}$ in Hank's Balanced Salt Solution (HBSS, GIBCO) supplemented with $1 \%$ Penicillin-Streptomycin, $0.2 \%$ type IV Collagenase and $0.05 \%$ type IV Elastase. In order to assess the quality of the smooth muscle cell preparation, immunofluorescence staining with a monoclonal antimouse $a$-smooth muscle actin antibody and a goat anti-mouse IgG coupled to Alexa-Fluor 488 as secondary antibody was performed and revealed that $95 \%$ of cells were positive for this specific biomarker.

\section{Isometric tension measurements}

Fresh distal rat PA rings were dissected from rat lungs. Tension measurements were performed exactly as previously described [22]. A 
Hiram R (2016) MAG-DHA, precursor of D-series resolvins, induces powerful resolution of various components of pulmonary hypertension induced by monocrotaline in rats

A
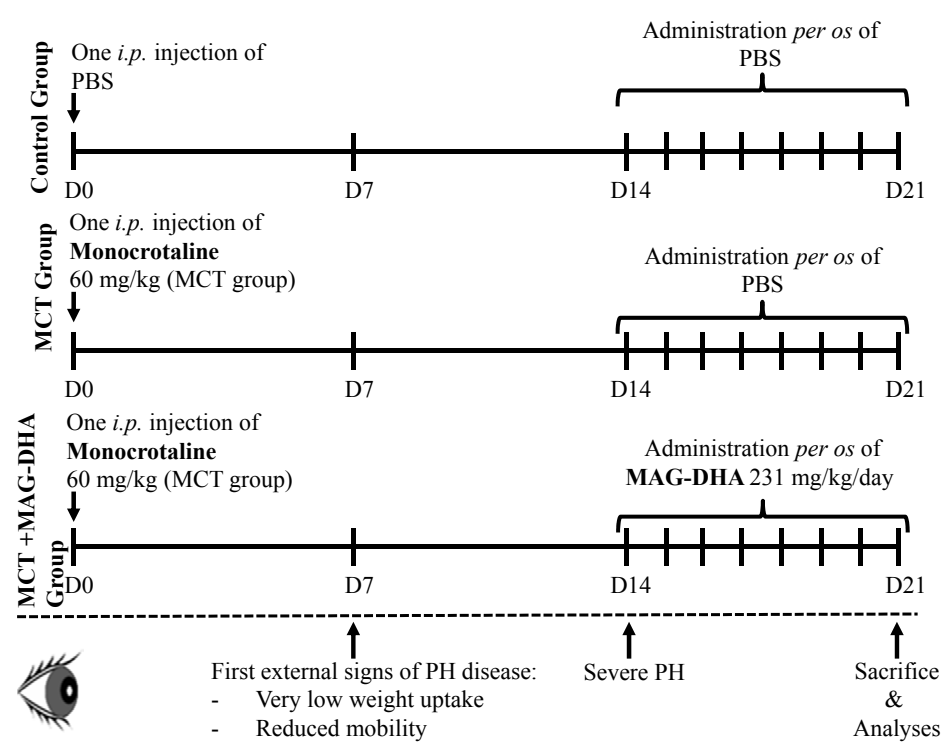

B

Lung histology at Day 14
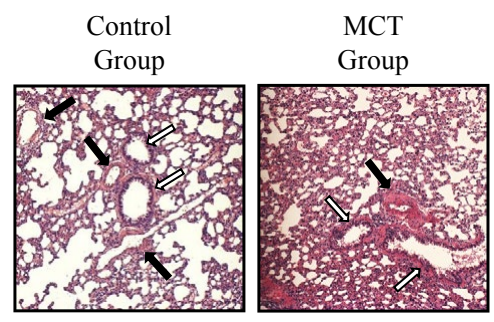

$\mathrm{MCT}+\mathrm{MAG}-\mathrm{DHA}$

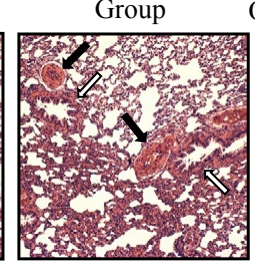

Quantitative analyses

Figure 1. Study design and histological analysis.

(A) Experimental design and treatments. Three groups of rats were studied: Control, MCT-treated and MCT + MAG-DHA-treated. At day 0 (D0), rats from the Control group were injected i.p. with PBS; rats from the two other groups were injected with $60 \mathrm{mg} / \mathrm{kg}$ of MCT. At D14, 2 rats from each group were sacrificed and lung tissues were resected, sectioned and stained with hematoxylin-eosin for histological analysis. From D14 to D21, rats from the Control and MCT groups received oral administration of PBS while the MCT + MAG-DHA group received $231 \mathrm{mg} / \mathrm{kg}$ MAG-DHA daily. At D21, all animals were sacrificed and tissues processed for comparative pulmonary artery isometric tension measurements, lung histological analysis, lung homogenate preparations and SMC isolation. (B) Histological analysis of lung tissue sections stained with hematoxylin-eosin from Control, MCT and MCT + MAG-DHA-treated groups as well as quantitative analyses of pulmonary artery thickness at day 14 , following the injection of MCT at day 0 . Black arrow point to pulmonary artery and white arrow to bronchi. Quantification is representative of 10 pulmonary arteries per lung.

basal tension of $0.6 \mathrm{~g}$ was applied to each ring. Pharmaco-mechanical responses induced by specific agonists, including $\mathrm{U}-46619$ and $\mathrm{PDBu}$ and $80 \mathrm{mM} \mathrm{KCl}$ were assessed on active tensions, using transducer systems coupled to Polyview software (Grass-Astro-Med Inc, West Warwick, RI) enabling to perform data acquisition and analysis. The amplitudes of maximum tensions were expressed for a given agonist concentration $(30 \mathrm{nM} \mathrm{U}-46619$ or $1 \mu \mathrm{M} \mathrm{PDBu})$ normalized to the control response to $80 \mathrm{mM} \mathrm{KCl}$ for each tested PA ring.

\section{SDS-PAGE and Western blot analyses}

Microsomal, cytosolic and nuclear fractions were prepared as previously described [10] using tissue homogenates prepared from pulmonary arteries of control and treated animals. Western blots were performed using specific antibodies against membrane and cytosolic proteins (TMEM16A, GPR32, HIF1- $\alpha$ and $\beta$-actin proteins) and phosphorylated isoforms of regulatory proteins of the contractile machinery (phospho-CPI-17 and phospho-MYPT-1) as well as pro-inflammatory markers (TNF- $\alpha$ and phospho-p65-NFkB). Blot immunostainings were revealed on Kodak film and subsequently digitized and analyzed using ImageJ software [23].

\section{Data presentation and statistical analyses}

Results are expressed as means \pm SEM, with $\mathrm{N}$ indicating the number of rats and $\mathrm{n}$ indicating the number of experiments performed or tissues that have been used. Statistical analyses were performed using a Student $t$ test or one-way ANOVA with Sigma Plot 12.0 and SPSS 14.0 software (SPSS-Science, Chicago, IL). The ANOVA was followed by Fisher's least significant difference (LSD) test. Differences were considered statistically significant $\left(^{*}\right)$ when $P<0.05$.

\section{Results}

\section{Experimental design and preliminary results}

The DHA metabolite Resolvin D1 has recently been shown to reduce inflammation markers and hyperreactivity in human pulmonary artery upon in-vitro treatment [17]. To strengthen these reports, the current 
Hiram R (2016) MAG-DHA, precursor of D-series resolvins, induces powerful resolution of various components of pulmonary hypertension induced by monocrotaline in rats

experimental series were performed on tissues from an in-vivo rat model of pulmonary hypertension. Control group rats were injected with PBS i.p. at day 0 (D0) while two other sets of rats (MCT group and MCT + MAG-DHA group) were injected i.p. with $60 \mathrm{mg} / \mathrm{kg}$ of MCT at day 0 and observed during 14 days.

From day 14 to day 21, the animals were administered either per os PBS (Control and MCT groups) or $231 \mathrm{mg} / \mathrm{kg}$ of MAG-DHA daily (MCT+MAG-DHA group) (Figure 1A). To assess the state and severity of pulmonary hypertension prior to MAG-DHA treatments, parallel sets of rats from each group were sacrificed at day 14 .

Figure 1B represents typical histological sections of rat lungs from Control, MCT and MCT + MAG-DHA groups at day 14. Quantitative analysis of pulmonary artery wall thickness revealed that at 14 days, MCT treatment induced a significant increase in wall thickness in comparison to rat pulmonary artery from the Control group.

The morphometry and histology of pulmonary arteries from the MCT + MAG-DHA group (MAG-DHA not yet administered) were identical to those from the MCT group at day 14.

\section{MAG-DHA reverses induction of right ventricular hypertrophy induced by monocrotaline}

From day 14 to day 21, the three groups of rats were treated as described above and in Figure $1 \mathrm{~A}$. The animals from the first two groups received PBS, while the animals from the third group received daily doses of MAG-DHA. All rats were sacrificed at day 21.

Figure 2A represents bar graphs of the Fulton's Index, calculated according to the equation linking right ventricular weight (RV) over left ventricular (LV) plus septum weight (S) and given by

\section{Fulton's Index $=$ RV $(\mathrm{g}) /(\mathrm{LV}+$ SEPTUM $)(\mathrm{g})$}

In comparison to control condition, MCT injection induced a significant increase in Fulton's Index, suggesting significant hypertrophy of the right ventricle due to MCT-triggered pulmonary hypertension in the microvessels. Daily ingestion of MAG-DHA for 7 days (D14 to D21) significantly reversed this hypertrophy with Fulton's index returning toward mean control values.

Figure $2 \mathrm{~B}$ displays the weight curve from day 0 to day 21 . The results indicate that control rats consistently gained weight from $400 \mathrm{~g}$ (day 0) to $495 \mathrm{~g}$ (day 21) on average. However, during the first fourteen days under MCT, rats had a lower weight gain (of approximately $25 \mathrm{~g}$ ). In contrast, rats treated with MAG-DHA for 7 days gained $50 \mathrm{~g}$, which was twice as much as the recorded growth of MCT-treated rats over the same period.

\section{MAG-DHA reverses the histological effects induced by MCT}

Twenty-one days after MCT injection, all rats were sacrificed and lungs were excised and samples fixed in $4 \%$ paraformaldehyde during $48 \mathrm{~h}$. Thin sections were subsequently prepared and stained with Hematoxylin-Eosin to identify nuclei and eosinophilic structures while Masson's Trichrome staining was used to differentiate muscle fibers from collagen. Figure 3 represents typical histological sections from the three groups of rats: control rats (untreated) (Figure 3A and B), MCT-treated rats (Fig. 3C and D), and MCT + MAG-DHA-treated rat (Figure 3E and F). Compared to control, lungs from MCT-treated rats displayed several histological differences as well as heterogeneity in parenchymal organization. Alveoli were larger although less numerous than in control condition. Pulmonary artery walls appeared to be
A

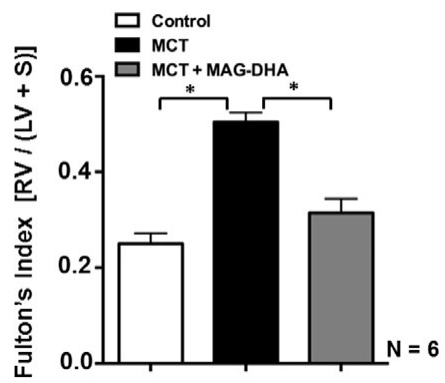

B

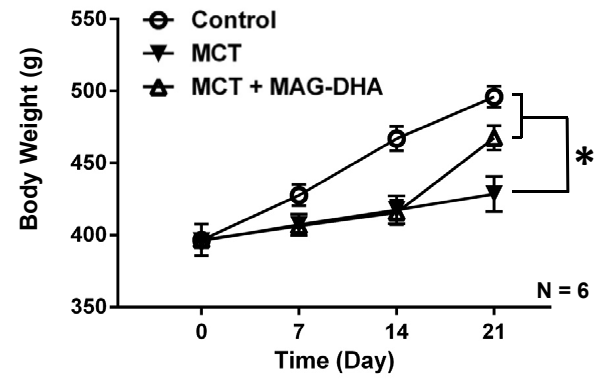

Figure 2. Effect of MCT and MAG-DHA on Fulton's index and body weight changes as a function of time

(A) The Fulton's index (ratio of right ventricular weight over left ventricular + septum weight) was calculated to assess right ventricular (RV) hypertrophy following MCT treatment and RV normalization following MAG-DHA treatment. (B) Body weight (g) expressed as a function of time (day) was measured in Control, MCT- and MCT + MAGDHA- treated rats. Results are expressed as the mean \pm s.e.m $\left(\mathrm{N}=6\right.$ rats per group) ${ }^{*} \mathrm{p}<$ $0.05)$.

hypertrophied in MCT condition suggesting an abnormal proliferation of smooth muscle cells in the media. The endothelial layer was also disorganized and hypertrophied. The lumen appeared to be closed when compared to the diameter observed in control pulmonary artery. Of note, 7-day treatment with MAG-DHA partially reversed the above histological changes initially induced by MCT injection. Consequently, following MAG-DHA treatment, the overall structure of lung tissues closely resembled that documented under control condition, suggesting a potential reversibility of the severe morphological and functional changes associated with MCT-induced PH.

MAG-DHA treatment reverses media hypertrophy and pulmonary artery hyper-responsiveness induced by MCT treatment

Figure 4A shows that MCT treatment induced a significant increase in media thickness with a mean thickness of $48 \pm 5 \mu \mathrm{m}$ compared to 12 $\pm 3 \mu \mathrm{m}$ observed in control rats. A 7-day treatment with MAG-DHA was sufficient to resolve and basically reverse the hypertrophy induced by MCT treatment, with a resulting mean media artery thickness of 20 $\pm 3 \mu \mathrm{m}$ following MAG-DHA administration. In conjunction with the histological analyses, this increase in pulmonary artery wall thickness is thus indicative of a proliferation of smooth muscle cells and an increase in the ability to develop an over-contractility and pressure.

Given the above, we next assessed the contractile and pharmacological properties of pulmonary arteries obtained from the various rat groups. After a 45-min equilibration period, pulmonary arteries were sequentially stimulated with $80 \mathrm{mM} \mathrm{KCl,} 30 \mathrm{nM} \mathrm{U}-46619$ 
Hiram R (2016) MAG-DHA, precursor of D-series resolvins, induces powerful resolution of various components of pulmonary hypertension induced by monocrotaline in rats

\section{Lung histology at Day 21}
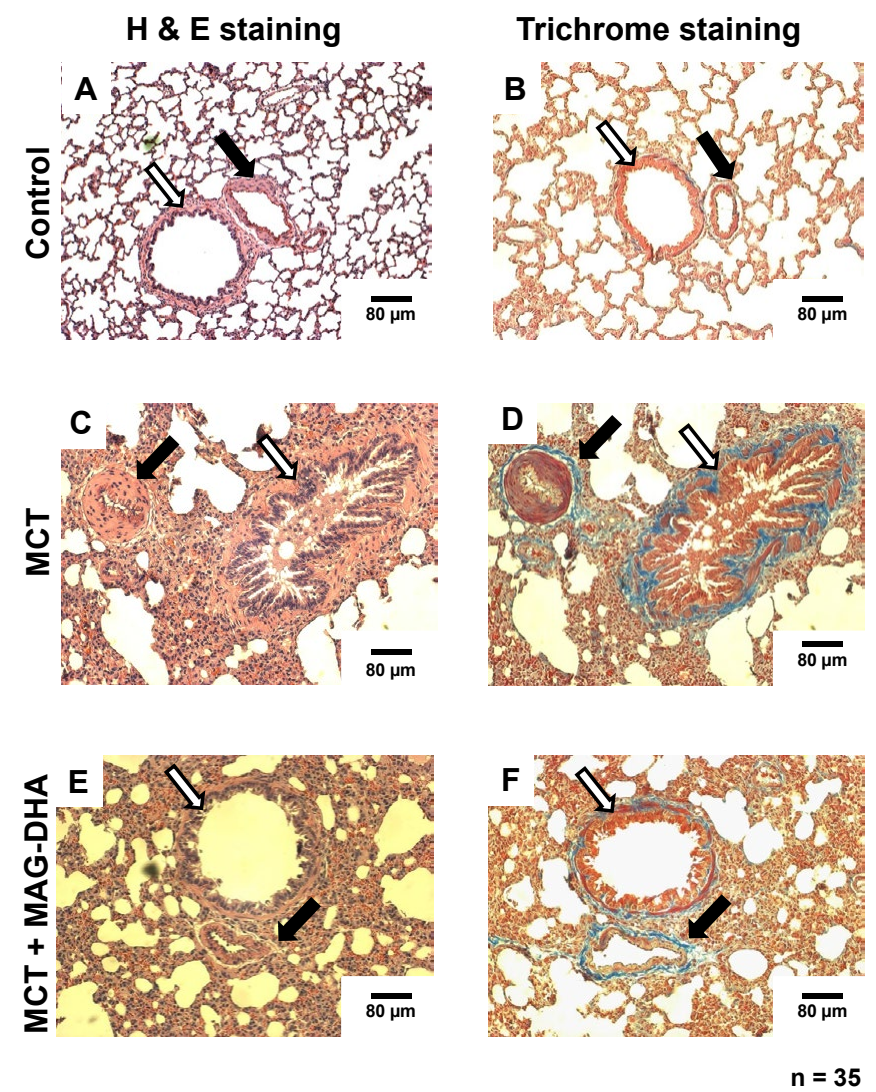

Figure 3. Histological analysis of rat lungs at day 21 under various experimental conditions. Photomicrographs of hematoxylin and eosin (H\&E)-stained (panels A, C and E) and Masson's Trichrome-stained (panels B, D and F) thin sections from rat lungs showing the morphological changes of medial thickness of the pulmonary arterial wall in tissues from Control, MCT- and MCT + MAG-DHA- treated rats. Black arrow $=$ Pulmonary artery. White arrow $=$ Bronchi. Scale Bar $=80 \mu \mathrm{m} .\left({ }^{*} \mathrm{n}=25, \mathrm{p}<0.05\right)$

and $1 \mu \mathrm{M}$ PDBu for 10 min respectively, followed by 45 min washouts periods after each stimulus. Figure $4 \mathrm{~B}$ depicts the relative amplitudes of the responses to all three stimuli. On average, pulmonary arteries from MCT-treated rats were significantly more reactive than those of the control group with tension developed by the MCT group reaching twice the values of the control group. Conversely, MAG-DHA normalized the contractile responses triggered by $\mathrm{KCl}, \mathrm{U}-46619$ and $\mathrm{PDBu}$, as well as reversed the over-reactivity developed by pulmonary arteries from the MCT-treated group (Figure 4B).

The phosphorylation levels of regulatory proteins of the contractile machinery were also measured. Figures $4 \mathrm{C}, \mathrm{D}$ and $\mathrm{E}$ reveal that two phosphoproteins (namely P-CPI-17 and P-MYPT-1) were enhanced by MCT treatment and that the phosphorylation ratio of P-CPI-17/CPI17 and P-MYPT-1/MYPT-1 was significantly increased in pulmonary arteries from MCT-treated rats compared to control condition (Figure $4 \mathrm{D}$ and $4 \mathrm{E})$.

MAG-DHA treatment following MCT injection consistently induced a significant reduction of these ratios, leading to mean values nearing those observed under control condition (Figure 4D and 4E). Pharmacological treatment regulates the expression of
biological markers of pulmonary hypertension

In pulmonary hypertension, HIF1- $\alpha$ is rapidly internalized into the
A

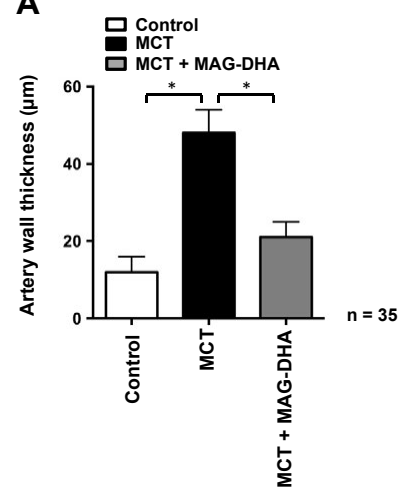

B

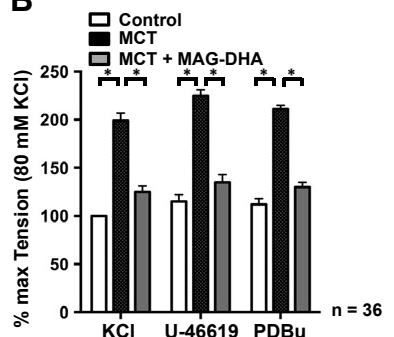

C

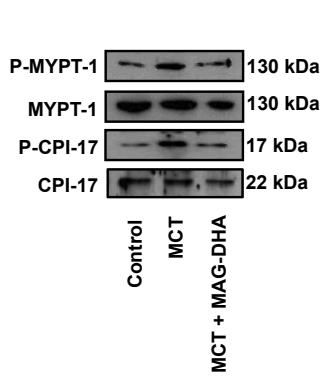

D

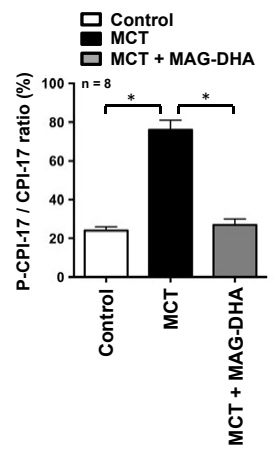

E

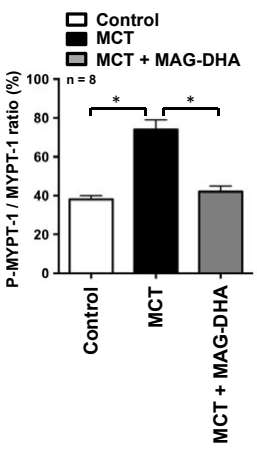

Figure 4. Effect of MAG-DHA on mean pulmonary artery wall thickness and contractility in MCT-treated rats. (A) Vascular wall thickness of rat pulmonary arteries under Control, MCT- or MCT + MAG-DHA- treated conditions was measured and expressed in $\mu \mathrm{m}\left({ }^{*} \mathrm{n}=\right.$ $35, \mathrm{P}<0.05$ ). (C) Representative recordings of the mechanical tension induced by $80 \mathrm{mM}$ $\mathrm{KCl}, 30 \mathrm{nM} \mathrm{U} 46619$ and $1 \mu \mathrm{M} \mathrm{PDBu}$ on fresh pulmonary arteries microdissected from rat lungs of Control, MCT- and MCT + MAG-DHA-treated animals $(* n=18, P<0.05$ ). Of note all mechanical tensions were normalized to the $\mathrm{KCl}$ responses, which in control condition resulted in an average tension of $0.32 \pm 0.03 \mathrm{~g}$. (C) Western blot analysis of lung homogenate fractions from Control, MCT- and MCT + MAG-DHA-treated rats using specific antibodies against Phospho-MYPT-1, MYPT-1, Phospho-CPI-17 and CPI-17. (D) Quantitative analysis of CPI-17 and (E) MYPT-1 phosphorylation levels expressed as a ratio of the total molecules. $(* \mathrm{n}=8, \mathrm{P}<0.05)$.

nucleus under hypoxic conditions in order to activate and up-regulate target genes that promote cell response to hypoxia while TMEM16A has been described as a biomarker of the disease because of its enhanced expression in the membrane of PASM cells [7]. In a previous study, we also demonstrated that MAG-DHA needs to be metabolized to exert its beneficial action, and that one of its metabolites, resolving D1 (RvD1), leads an enhanced expression of GPR32 [17], previously identified as the $\mathrm{RvD1}$ receptor [24]. In order to further assess the manner in which MAG-DHA may counteract the effects of MCT treatment on the expression of these key biological markers, PASM cell lysates were fractionated using differential ultracentrifugations from which microsomal fractions were used to detect expression of membrane proteins (TMEM16A and GPR32) while cytosolic fractions were used to detect the expression of HIF1- $\alpha$. Figure $5 \mathrm{~A}$ and $5 \mathrm{~B}$ demonstrate that compared to control rats, HIF1- $\alpha$ was highly detected in the nucleus of PASM cells of MCT-treated rats, whereas MAG-DHA treatment significantly reduced the expression of HIF1- $\alpha$ to a level similar to the control condition. TMEM16A protein detection (Figure 5A and 5C) was also enhanced under MCT condition when compared to controls, while addition of MAG-DHA significantly reversed this overexpression toward control values. In contrast, GPR32 protein (Figure $5 \mathrm{~A}$ and 5D) was expressed at low levels in both control and MCT 
A

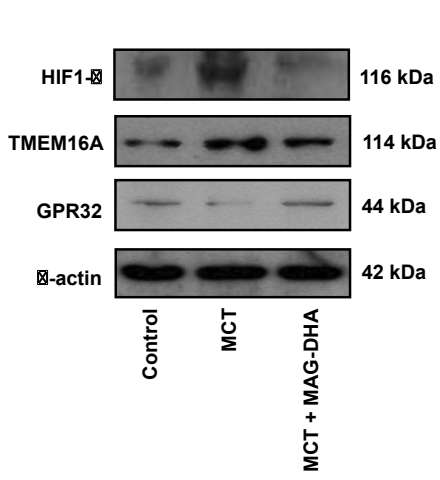

C

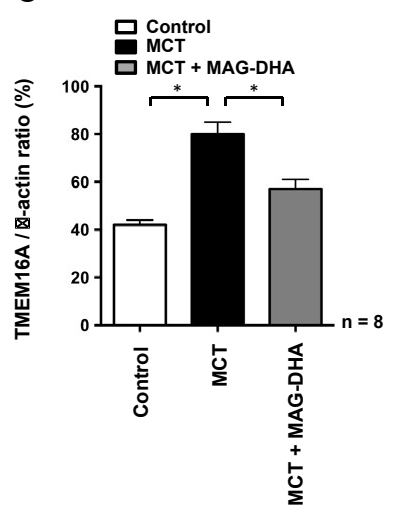

B

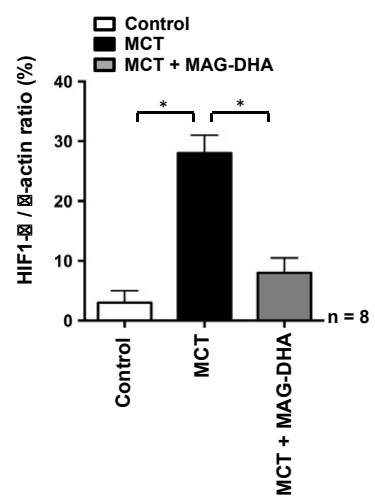

D

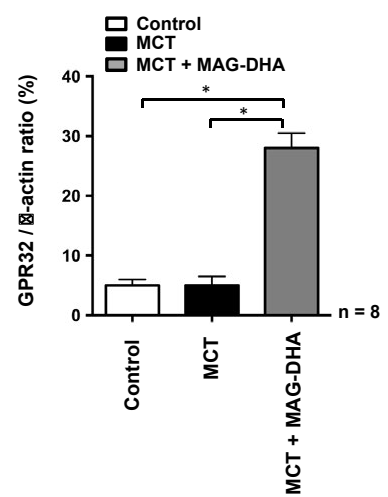

Figure 5. Effect of MAG-DHA on HIF1- $\alpha$, TMEM16 and GPR32 protein detection

(A and B) Western blot analyses were performed using antibodies against HIF1- $\alpha$ on cytosolic fractions of cell lysates isolated from pulmonary arteries of rats treated under Control, MCT and MCT + MAG-DHA conditions. Relative detection of HIF1- $\alpha$ was expressed as a function of cytosolic $\beta$-actin (B). (A and C) Western blot analyses were performed using antibodies against TMEM16A from microsomal fractions of cell lysates isolated under the same conditions as described above. Results are expressed as the relative TMEM16A / $\beta$-actin ratio. (A and D) Western blot analyses using antibodies against the GPR32 receptor were performed from microsomal fractions of cell lysates from pulmonary arteries of rats treated under the same conditions as described above $\left({ }^{*} \mathrm{n}=8, \mathrm{P}<0.05\right)$.

conditions. However, MAG-DHA treatment in MCT rats induced a large increase in GPR32 detection in the microsomal fraction of PASM cells. These results confirm that MCT treatment induced a net increase in pulmonary hypertension biomarkers while 7 days of MAG-DHA treatment resulted in a significant decreased detection of HIF1- $\alpha$, TMEM16A and GPR32 (RvD1 receptor). Thus MAG-DHA appears to resolve the MCT-induced effects on histological and biochemical markers via the action of its D-series metabolites as attested by the enhanced expression of GPR32 [10].

\section{Inflammatory markers are also up-regulated in pulmonary hypertension}

Given that pulmonary arteries from patients with thromboembolic $\mathrm{PH}$ have been reported to display a number of enhanced proinflammatory markers [25], including the signaling molecules TNF- $\alpha$, COX-2 and STAT3 [26,27], these latter biomarkers were also investigated in the current experimental model. TNF- $\alpha$ has been shown to reduce prostacyclin production and to increase other pro-inflammatory cytokines secretion in pulmonary artery tissues [28]. As a key modulator of the aggravation of pulmonary artery wall hypertrophy, the expression of TNF- $\alpha$ has been investigated in the present study. Our results show that, compared to the control

condition, MCT treatment induced an increase in TNF- $\alpha$ (Figure $6 \mathrm{~A}$ and $6 \mathrm{~B}$ ), COX-2 (Figure 6A and 6C) and STAT3 (Figure 6A and 6D) expression levels, whereas MAG-DHA treatment lowered the expression level of these pro-inflammatory biomarkers. Moreover, when TNF- $\alpha$, COX-2 or STAT3 (which is activated by IL-6) are concomitantly increased, these biomarkers stimulate various signaling

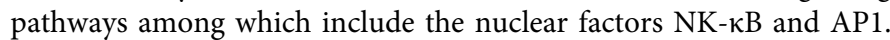
These two nuclear transcription factors have previously been shown to play an important role in stimulating the expression of inflammatory cytokines involved in pulmonary artery remodeling, thus increasing the severity of PA disease [6]. Results obtained in nuclear fractions show that compared to control, MCT treatment induced an increase in the phosphorylation level of NF- $\kappa B$, while 7-day treatment with MAGDHA significantly reduced this level, leading to values approximating those observed in controls (Figure 7A and 7B). Data also show that compared to the control condition, MCT treatment increased the phosphorylation levels of both c-Fos and c-Jun, the levels of which were subsequently normalized following MAG-DHA treatment (Figure 7A, $7 \mathrm{C}$ and $7 \mathrm{D})$. Together these data suggest that MAG-DHA treatments are able to normalize most of the biochemical and functional changes induced by MCT.

\section{Discussion}

In the present report, we investigated the in-vivo ability of MAGDHA to resolve inflammatory biomarkers and pulmonary artery over-
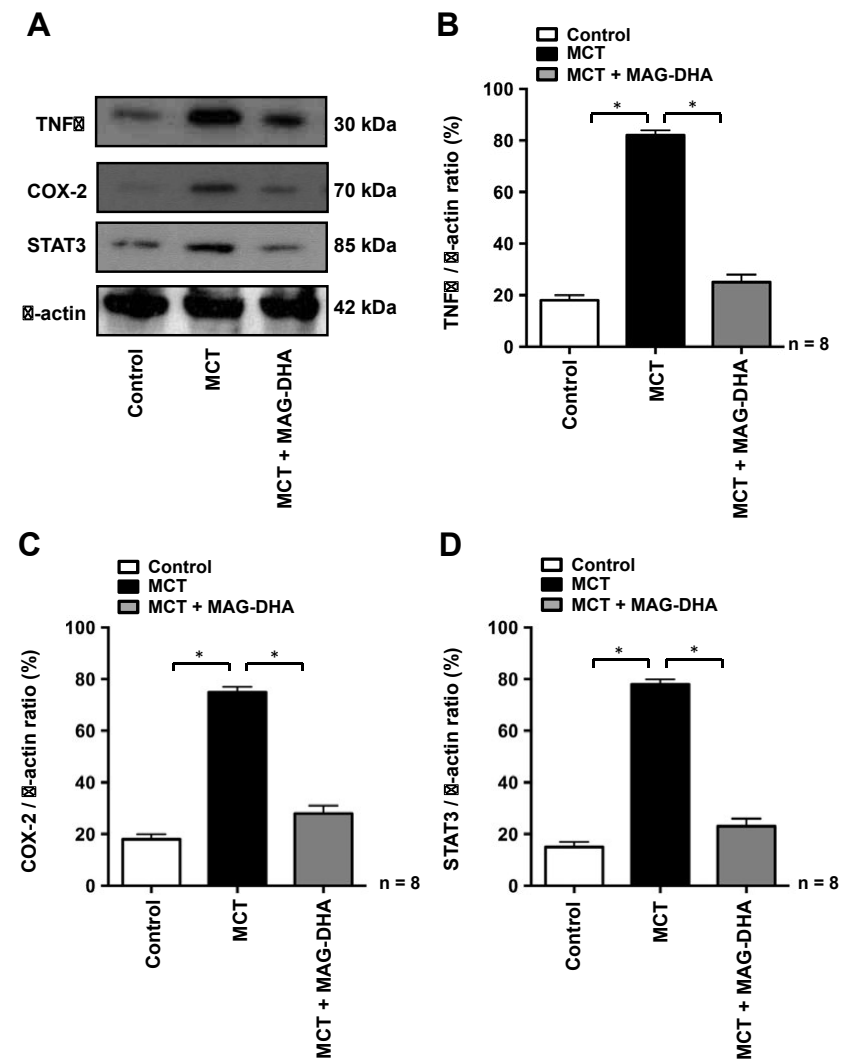

Figure 6. Resolving effects of MAG-DHA on the detection of inflammatory biomarkers.

(A) Western blot analyses were performed using antibodies against TNF- $\alpha$, COX-2, STAT3 and $\beta$-actin from rat pulmonary artery homogenates. (B), (C) and (D) represent the respective quantitative analyses of the expression level of these pro-inflammatory markers in control, MCT or MCT + MAG-DHA-treated conditions, expressed as a ratio with $\beta$-actin. $(* \mathrm{n}=8, \mathrm{P}<0.05)$. 
Hiram R (2016) MAG-DHA, precursor of D-series resolvins, induces powerful resolution of various components of pulmonary hypertension induced by monocrotaline in rats

A
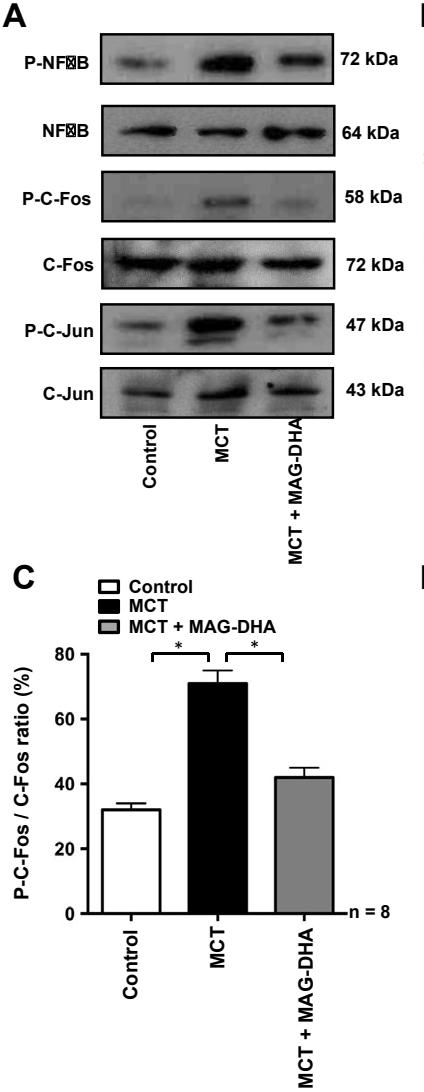

B

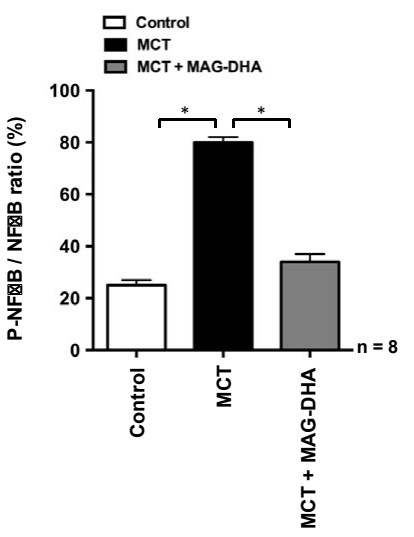

D
Control
MCT + MAG-DHA
MCT * ${ }_{*}$

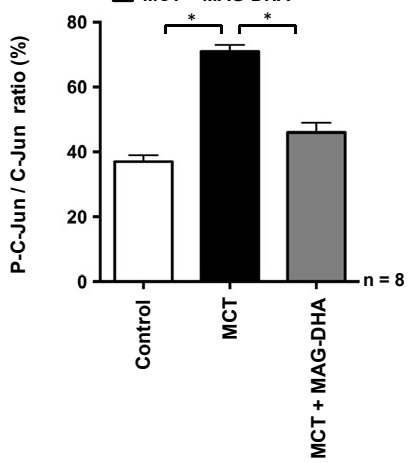

Figure 7. Effects of MAG-DHA on the detection of three pro-inflammatory nuclear factors. (A) Western blot analyses were performed on nuclear fractions from homogenates, using specific antibodies against $\mathrm{NF \kappa B}, \mathrm{C}$-Fos, C-Jun and their respective phosphorylated forms in control, MCT or MCT + MAG-DHA-treated groups. Relative quantitative analyses were

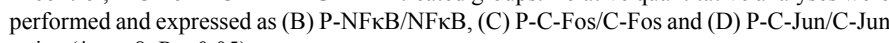
ratios $(* n=8, P<0.05)$.

reactivity as well as its ability to curb the abnormal inflammatory status, increased artery vasoconstriction and PASM cell over-reactivity in an in-vivo rat model of MCT-induced pulmonary hypertension. Fourteen days after the MCT injection, rats developed several characteristics of pulmonary artery hypertension as indicated by Fulton's index and histological assessment, as well as the observed weight loss. After a 7-day MAG-DHA treatment, the Fulton's index returned to normal values and the mean weight loss was largely corrected. MAG-DHA is neutral lipid which is very well absorbed by tissues [29] and becomes a DHA donor upon the action of MAG-lipase and the precursor of several omega-3 derivatives such as resolvins and protectins upon the action of 15-LOX and 5-LOX [10]. Resolvins have been reported to reduce pro-inflammatory markers $[17,30]$, namely TNFa and IL$1 \beta$, and to prevent systemic hypertension in rats [31]. Furthermore, RvD1, A trihydroxylated DHA derivative has recently been reported to reverse the pharmacological reactivity and $\mathrm{Ca}^{2+}$ sensitivity induced by ET-1 and IL-6 in human pulmonary artery [17].

Our current data further demonstrate that in a well characterized model of pulmonary hypertension, MAG-DHA was able to reverse the pathological remodeling of the pulmonary artery wall, as well as the concomitant pharmacological over-reactivity. Moreover, specific inflammatory biomarkers such as TNFa, COX-2 and STAT3 were significantly reduced to prevent the typical underlying events related to the pathophysiology of the disease.

\section{Induction of PH by Monocrotaline}

Monocrotaline (MCT) is a pyrrolizidine alkaloid from Crotalaria spectabilis which is activated in the liver into monocrotaline pyrrole and transferred to the lung where it induces chronic pneumotoxic effects $[21,32]$ characterized by increased synthesis of pro-inflammatory cytokines leading to macroscopic and microscopic changes such as parenchymal infiltration fibrosis, PASM cell proliferation and pulmonary artery wall hypertrophy [32,33]. Severe pulmonary hypertension is subsequently observed and is consistent with an increased vasoconstriction, related to an enhanced blood pressure (confirmed by an increased expression of markers of hypoxia such as HIF-1 $\alpha$ ) and induced right ventricular hypertrophy which can trigger heart failure and sudden death $[33,34]$.

In the adventitia constituting the outer layer of the pulmonary artery, enhanced pro-inflammatory markers appear as a common denominator to multiple events which are in turn triggered and contribute to the severity of $\mathrm{PH}$. It has been reported that theaccumulation of cytokines and chemokines such as TNF- $\alpha$, IL- $1 \beta$, and IL-6 is a key phenomenon in the genesis of the disease [6]. These agents control and regulate a wide network of intracellular signalling pathways such as NFkB, STAT3, c-Fos and c-Jun dependent pathways [33], that are directly involved in the activation and expression of genes such as COX-2 [28], known to initiate cell proliferation, extracellular matrix disorganization, abnormal vascular growth and remodeling [26].

Much of the current medications and therapeutic strategies used to treat patients primarily target endothelial dysfunctions related to $\mathrm{PH}$, in order to facilitate PASM cell relaxation and pulmonary artery dilatation [10]. However, there is no eradication of the disease; moreover, specific lung inflammation has paradoxically not been targeted, whether it is to change the course or to cure $\mathrm{PH}$. Consequently, there is a clinical gap in terms of proposing new therapeutic strategies and in designing specific clinical studies.

\section{Resolving effects of MAG-DHA}

N-3 PUFAs have been demonstrated to trigger anti-inflammatory effects in various diseases such as rheumatoid arthritis [35], neurodegenerative diseases [36], asthma [23] and more recently, pulmonary hypertension [16]. In the present study, we uncovered the ability of MAG-DHA to alleviate inflammation, pulmonary artery remodeling and pharmaco-mechanical over-reactivity using an in-vivo rat model of MCT-induced pulmonary hypertension. The monoacylglyceride form of DHA increases the bioavailability of this long chain omega- 3 fatty acid, thus enabling to overcome the limited half-life of the negatively charged compound. Furthermore, the beneficial effects of DHA have been reported in several studies aimed at the prevention of cardiovascular diseases [13,22,38]. Hence, our present study depicts an original approach to investigate the curative role of DHA or one of its metabolites in $\mathrm{PH}$.

MAG-DHA resolves inflammation in MCT rats: Our data reveal that curative treatment with MAG-DHA was able to reverse the MCTinduced increase in TNF- $\alpha$ expression in rat lungs. Furthermore, as previously proposed in a recent report which investigated the role of another N-3 PUFA, namely MAG-DPA [16], and regarding the cascade of the various genes involved in artery wall remodeling and PASM cell dysfunction, NFKB appears to be of key importance as a mediator from which MAG-DHA or its metabolites, such as D-series resolvins, may trigger beneficial effects through specific receptors, notably the RvD1 
Hiram R (2016) MAG-DHA, precursor of D-series resolvins, induces powerful resolution of various components of pulmonary hypertension induced by monocrotaline in rats

receptor GPR32. Of note, the expression of this specific receptor was largely enhanced following MAG-DHA treatment (Figure 5D). This observation confirms a previous report suggesting that GPR32 is induced by the presence of omega-3 derivatives in various tissues [17].

Further studies are nonetheless needed to confirm the central role of the NF- $\mathrm{kB}$ signaling pathway and to assess the expression of specific gene products and/or to describe the evolution of $\mathrm{PH}$ in MCT and hypoxic rats with a knocked-out NF- $\mathrm{B}$ gene. The regulation of gene activation involved in the remodeling of the pulmonary artery represents another issue that needs to be addressed to curb the worsening of $\mathrm{PH}$.

Curative effects of MAG-DHA on pulmonary artery morphology and reactivity: In the present study, MCT treatment induced significant modifications in lung histology when compared to thin sections from control rats. In addition to increased artery thickness, pulmonary arteries became over-reactive in response to pharmacological stimuli. While the mechanisms by which MAG-DHA interacts with the contractile machinery remain unclear, recent studies have nevertheless proposed relevant modes of action that could explain the cellular signals that are activated or inhibited by DHA and thus potentially participate in its beneficial effects $[35,38,39]$. Of these include the aforementioned trihydroxylated DHA metabolites such as RvD1-RvD4 [24]. The RvD1 receptor or GPR32 has indeed been described to be overexpressed when arteries are treated with RvD1 [17]. Moreover, GPR32 has been reported to be a key receptor by which RvD1 can control expression of miRNAs to activate or inhibit expression of anti-inflammatory or proinflammatory agents, respectively [19]. Our data revealed that GPR32 detection was lowered in MCT rat lung artery, whereas MAGDHA treatment enhanced the expression of GPR32, thus suggesting that RvD1 may have been produced under this condition in order to interact with intracellular signals to resolve the inflammatory-induced dysfunctions and ultimately normalize pulmonary artery reactivity. MAG-DHA could be further investigated as a potential preventive or curative treatment in the resolution of the remodeling events in vascular diseases.

\section{Limitations of the study}

The design of the present study differs from the usual investigations performed in our laboratory regarding the effect of N-3 PUFAs on an in-vivo rat model of MCT-induced $\mathrm{PH}$, because of the curative nature of the current study. The data reported herein offer valuable mechanistic insight into the putative modes of action of MAG-DHA, or its derivatives, on pulmonary artery inflammation, wall remodeling and pharmacological reactivity. However, the precise signaling pathways remain to be ascertained. Consequently, complementary studies will be required to delineate key proteins and secondary messengers involved in the regulation and resolution of inflammation in this model. While the influence of N-3 PUFAs on miRNA regulation has recently been assessed, the exact signaling pathways remain to be identified [19]. Another limitation of our study was the absence of systolic pressure measurements in pulmonary artery in the absence and presence of MAG-DHA; this issue will be the topic of an ancillary project.

\section{Short term perspective}

It would be relevant to perform the detection of resolvins in blood and lung tissues in order to ascertain whether MAG-DHA could play its beneficial effects via its bioactive metabolites such as D-series resolvins, as suggested in a previous in-vitro study on human pulmonary artery [17]. However it is important to note that Resolvins D1, D2 and D3 usually display short life-times. Thus, it may prove difficult to measure their respective plasma concentrations in the in-vivo rat model used.

\section{Conclusion}

The present study attests that MAG-DHA is able to reverse invivo pulmonary hypertension induced by MCT-treatment in a rat model. Expression levels of specific proteins involved in inter- and intracellular signalling (TNFa, NFאB, STAT3), remodeling (COX2, c-Fos, c-Jun) and mechanical reactivity (P-CPI-17, P-MYPT-1) were moreover normalized following MAG-DHA curative treatment. Altogether, the present data, as well as the demonstrated bioavailability and low toxicity of its derivatives $[17,18]$, suggest that MAG-DHA could represent a potential new therapeutic precursor in resolving inflammatory status in pulmonary hypertension.

\section{Acknowledgements}

We thank Mr. Pierre Pothier for critical review of the manuscript. This work was supported by a grant from the Heart and Stroke Foundation of Canada/Quebec to Eric Rousseau and Caroline Morin. Roddy Hiram is the recipient of a studentship from the Health Respiratory Training Program of Quebec supported by the CIHR.

\section{Conflicts of interest}

The authors have declared no conflicts of interests. Samuel Fortin is the owner of SCF Pharma, which has an exclusive worldwide license of the patented compositions and uses of MAG-DHA.

\section{References}

1. Hoeper MM (2009) The new definition of pulmonary hypertension. Eur Respir J 34 790-791. [Crossref]

2. Nauser TD, Stites SW (2001) Diagnosis and treatment of pulmonary hypertension. $\mathrm{Am}$ Fam Physician 63: 1789-1798. [Crossref]

3. Brown LM, Chen H, Halpern S (2011) Delay in recognition of pulmonary arterial hypertension: factors identified from the REVEAL Registry. Chest 140:19.

4. Simonneau G, Robbins IM, Beghetti M, Channick RN, Delcroix M, et al. (2009) Updated clinical classification of pulmonary hypertension. J Am Coll Cardiol 54: S4354. [Crossref]

5. Stewart DJ, Levy RD, Cernacek P, Langleben D (1991) Increased plasma endothelin-1 in pulmonary hypertension: marker or mediator of disease? Ann Intern Med 114: 464 469. [Crossref]

6. Price LC, Wort SJ, Perros F, Dorfmüller P, Huertas A, et al. (2012) Inflammation in pulmonary arterial hypertension. Chest 141: 210-221. [Crossref]

7. Forrest AS, Joyce TC, Huebner ML, Ayon RJ, Wiwchar M, et al. (2012) Increased TMEM16A-encoded calcium-activated chloride channel activity is associated with pulmonary hypertension. American J Physiol - Cell Physiol. 303: C1229-C1243.

8. Rodat-Despoix L, Aires V, Ducret T, Marthan R, Savineau JP, et al. (2009) Signalling pathways involved in the contractile response to 5 -HT in human pulmonary artery. European Respiratory Journal. 34: 1338-1347.

9. Lincoln TM (2007) Myosin Phosphatase Regulatory Pathways: Different Functions or Redundant Functions. Circulation Research. 100:10-12.

10. Hoeper MM, Bogaard HJ, Condliffe R, Frantz R, Khanna D, et al. (2013) Definitions and diagnosis of pulmonary hypertension. $J$ Am Coll Cardiol. 62: D42-50.

11. Galiè N, Corris PA, Frost A, Girgis RE, Granton J, et al. (2013) Updated treatment algorithm of pulmonary arterial hypertension. J Am Coll Cardiol. 62: D60-72.

12. Kähler CM, Colleselli D (2006) Pulmonary arterial hypertension associated to connective tissue diseases. Rheumatology. 45:11111-11113.

13. Andrade PM (2007) Effects of the fish-oil supplementation on the immune and inflammatory responses in elite swimmers. Prostaglandins Leukot Essent Fatty Acids 77:139-45.

14. Morin C, Sirois M, Echave V, Gomes MM, Rousseau E (2008) EET displays anti- 
Hiram R (2016) MAG-DHA, precursor of D-series resolvins, induces powerful resolution of various components of pulmonary hypertension induced by monocrotaline in rats

inflammatory effects in TNF-alpha stimulated human bronchi: putative role of CPI-17. Am J Respir Cell Mol Biol 38: 192-201. [Crossref]

15. Morin C, Fortin S, Rousseau E (2012) Docosahexaenoic acid monoacylglyceride decreases endothelin-1 induced $\mathrm{Ca}^{2+}$ sensitivity and proliferation in human pulmonary arteries. Am J Hypertens 25: 756-763. [Crossref]

16. Morin C, Hiram R, Rousseau E, Blier P, Fortin S (2014) Docosapentaenoic acid monoacylglyceride reduces inflammation and vascular remodeling in experimental pulmonary hypertension. Am. J. Physiol. Heart and Circulatory Physiology. 307: H574-86.

17. Hiram R, Rizcallah E, Sirois C, Sirois M, Morin C, et al. (2014) Resolvin D1 reverses reactivity and $\mathrm{Ca} 2+$ sensitivity induced by ET-1, TNF-a and IL-6 in human pulmonary artery. Am. J. Physiol. Heart and Circulatory Physiology.

18. Fortin S (2012) Compositions comprising polyunsatured fatty acids monoglycerides or derivatives thereof, and uses thereof. US patents. 819690 and 8222295 .

19. Recchiuti A, Krishnamoorthy S, Fredman G, Chiang N, Serhan CN (2011) MicroRNAs in resolution of acute inflammation: identification of novel resolvin D1-miRNA circuits. FASEB J 25: 544-60.

20. LALICH JJ, MERKOW L (1961) Pulmonary arteritis produced in rat by feeding Crotalaria spectabilis. Lab Invest 10: 744-750. [Crossref]

21. Huxtable RJ (1990) Activation and pulmonary toxicity of pyrrolizidine alkaloids Pharmacol Ther 47: 371-389. [Crossref]

22. Mozaffarian D, Wu JH (2011) Omega-3 fatty acids and cardiovascular disease: effects on risk factors, molecular pathways, and clinical events. J Am Coll Cardiol 58: $2047-$ 2067. [Crossref]

23. Morin C, Sirois M, Echave V, Rizcallah E, Rousseau E (2009) Relaxing effects of 17(18)-EpETE on arterial and airway smooth muscles in human lung. American Journal of Physiology 296: L130-L139.

24. Xu ZZ, Zhang L, Liu T, Park JY, Berta T, et al. (2010) Resolvins RvE1 and RvD1 attenuate inflammatory pain via central and peripheral actions. Nat Med 16: 592-597. [Crossref]

25. Zabini D, Heinemann A, Foris V, Nagaraj C, Nierlich P, et al. (2014) Comprehensive analysis of inflammatory markers in chronic thromboembolic pulmonary hypertension. Eur Resp J. 44: 951-962.

26. Fredenburgh LE, Ma J, Perrella MA (2009) Cyclooxygenase-2 inhibition and hypoxia- induced pulmonary hypertension: effects on pulmonary vascular remodeling and contractility. Trends Cardiovasc Med 19: 31-37. [Crossref]

27. Groth A, Vrugt B, Brock M, Speich R, Ulrich S, et al. (2014) Inflammatory cytokines in pulmonary hypertension. Respiratory Research. 15:47.

28. Itoh A, Nishihira J, Makita H, Miyamoto K, Yamaguchi E, et al. (2003) Effects of IL-1beta, TNF-alpha, and macrophage migration inhibitory factor on prostacyclin synthesis in rat pulmonary artery smooth muscle cells. Respirology. 8: 467-472.

29. Fortin S (2011) Polyunsaturated fatty acid monoglycerides, derivatives, and uses thereof, CA2672513, 2008, CA2677670, 2010, US8119690, 2011.

30. Hiram R, Rizcallah E, Marouan S, Sirois C, Sirois M, et al. (2015) Resolvin E1 normalizes contractility, $\mathrm{Ca} 2+$ sensitivity and smooth muscle cell migration rate in TNF-a- and IL-6-pretreated Human Pulmonary Arteries. Am. J. Physiol: Lung Cell and Mol Physiol.

31. Morin C, Rousseau E, Blier PU, Fortin S (2015) Effect of docosahexaenoic acid monoacylglyceride on systemic hypertension and cardiovascular dysfunction. $\mathrm{Am} \mathrm{J}$ Physiol Heart Circ Physiol 309: H93-93H102. [Crossref]

32. Wilson DW, Segall HJ, Pan LC, Lamé MW, Estep JE, et al. (1992) Mechanisms and pathology of monocrotaline pulmonary toxicity. Crit Rev Toxicol 22: 307-325. [Crossref]

33. Wang Q, Zuo XR, Wang YY, Xie WP Wang $H$, et al. (2013) Monocrotaline-induce pulmonary arterial hypertension is attenuated by TNF-a antagonists via the suppression of TNF-a expression and NF-?B pathway in rats. Vascul Pharmacol 58: 71-77.

34. Kay JM, Heath D (1969) Crotalaria spectabilis: The Pulmonary Hypertension Plant. Thomas, Springfield, IL.

35. Calder PC (2006) n-3 polyunsaturated fatty acids, inflammation, and inflammatory diseases. Am J Clin Nutr 83: 1505S-1519S. [Crossref]

36. Eckert GP, Lipka U, Muller WE (2013) Omega-3 fatty acids in neurodegenerative diseases: focus on mitochondria. Prostaglandins Leukot Essent Fatty Acids 88: 105-114.

37. Reagan-Shaw S, Nihal M, Ahmad N (2008) Dose translation from animal to human studies revisited. FASEB J 22: 659-661. [Crossref]

38. Swanson D, Block R, Mousa SA (2012) Omega-3 fatty acids EPA and DHA: health benefits throughout life. Adv Nutr 3: 1-7. [Crossref]

39. Uddin M, Levy BD (2011) Resolvins: natural agonists for resolution of pulmonary inflammation. Prog Lipid Res 50: 75-88. [Crossref]

Copyright: (C2016 Hiram R. This is an open-access article distributed under the terms of the Creative Commons Attribution License, which permits unrestricted use, distribution, and reproduction in any medium, provided the original author and source are credited. 